\title{
THE PERFECT-RECONSTRUCTION QMF BANK: NEW ARCHITECTURES, SOLUTIONS, AND OPTIMIZATION STRATEGIES
}

\author{
P. P. Vaidyanathan and Phuong-Quan Hoang \\ Department of Electrical Engineering \\ California Institute of Technology \\ Pasadena, CA 91125
}

\begin{abstract}
In this paper, a scheme for perfect reconstruction in $\mathrm{M}$ channel, maximally decimated QMF banks is first presented, for arbitrary $M$. The solutions are such that the analysis and synthesis filters are FIR and of the same length. Based on the theory, lattice structures for the two-channel case are derived, which offer an efficient design as well as implementation procedure for two-channel perfect reconstruction systems. Such lattice implementations are robust in the sense that the perfect-reconstruction property is preserved inspite of coefficient quantization.
\end{abstract}

\section{INTRODUCTION}

We consider the $M$ channel quadrature mirror filter bank shown in Fig. 1. Here $H_{k}(z)$ are the analysis filters, which split the incoming signal into $M$ adjacent frequency-bands. The subband signals are maximally decimated (i.e., decimated by $M$ ) and transmitted (after encoding). The received signals are interpolated (upgoing arrows), and recombined after passing through the synthesis filters $F_{k}(z)$. The reconstructed signal $\hat{x}(n)$ is related to the input signal $x(n)$ by the transform domain relation

$$
\hat{X}(z)=\sum_{l=0}^{M-1} X\left(z W^{-l}\right) \sum_{k=0}^{M-1} H_{k}\left(z W^{-l}\right) F_{k}(z) / M .
$$

The terms in (1) corresponding to $l \neq 0$ represent the effects of aliasing. If aliasing-distortion is canceled by appropriate choice of synthesis filters, the result is a time-invariant system with transfer function $T(z)=\hat{X}(z) / X(z)=\sum_{k=0}^{M-1} F_{k}(z) H_{k}(z) / M$ which is called the distortion transfer function. If $T(z)$ is allpass, there is no amplitude distortion; if $T(z)$ is a linear-phase FIR function, there is no phase distortion. Finally, if $T(z)=c z^{-n_{0}}$ which is a pure delay, then all distortions are absent, and we have perfect reconstruction feature (abbreviated PRF), i.e.,

$$
\hat{x}(n)=c x\left(n-n_{0}\right) .
$$

For the $M=2$ case, alias-cancelation techniques are well-known [1]-\{3], and for arbitrary $M$ approximate (but very useful) techniques for alias-calcelation are known $[7],[8]$. Smith and Barnwell have recently presented [4] a perfect reconstruction technique for the $M=2$ case, based on certain crucial properties of FIR half-band filters.

Recently, we outlined a technique [11] for perfect reconstruction in $M$ channel maximally decimated QMF banks, for arbitrary $M$. Even though the existence of perfect reconstruction systems has been known earlier [9], the method in [11] has the following features: all the analysis and synthesis filters $H_{k}(z)$ and $F_{k}(z)$ are FIR filters of the same length, and a very simple closed form expression is available for $F_{k}(z)$ in terms of $H_{k}(z)$. Moreover, arbitrary stopband attenuations for $H_{k}(z)$ can be achieved. In addition, our methods reduce to the Smith-Barnwell technique [4] for $M=2$. No inversion of transfer matrices is required anywhere in the process.

Since this paper is an application of a result in [11], we summarize this result here: first, note that any transfer function $H_{k}(z)$ can always be uniquely expressed as $H_{k}(z)=\sum_{l=0}^{M-1} z^{-l} E_{k l}\left(z^{M}\right)$ which is called the polyphase decomposition [2], and $E_{k l}(z)$ are the polyphase components. If we express each analysis filter $H_{k}(z)$ in this form, we can define an $M \times M$ matrix $\mathbf{E}(z)=\left[E_{k l}(z)\right]$ of polyphase components. In a similar manner, each synthesis filter can be expressed as $F_{k}(z)=\sum_{l=0}^{M-1} z^{-(M-1-l)} R_{l k}\left(z^{M}\right)$, thereby defining the $M \times M$ matrix $\mathbf{R}(z)$. Accordingly, Fig. $2(\mathrm{a})$ is an equivalent structure for Fig. 1. The decimators and interpolators can be relocated based on standard identities [2], to obtain Fig. 2(b).

If $\mathbf{E}(z)$ and $\mathbf{R}(z)$ satisfy $\mathbf{R}(z) \mathbf{E}(z)=c z^{-n_{1}} \mathbf{I}$ where $n_{1}$ is an arbitrary integer, then it is easily verified that (2) holds with $n_{0}=M-1+n_{1} M$. This requires that $\mathbf{R}(z)$ be of the form $c z^{-n_{1}} \mathbf{E}^{-1}(z)$. If $H_{k}(z)$ are FIR then $E_{k l}(z)$ are FIR, but $\mathbf{E}^{-1}(z)$ in general has IIR entries of high order, which may even represent unstable filters. Such matrix inversion and the associated problems can be avoided if $\mathbf{E}(z)$ is unitary on the unit circle. This property means that $\mathbf{E}^{\dagger}\left(e^{j \omega}\right) \mathbf{E}\left(e^{j \omega}\right)=\beta \mathbf{I}$ for all $\omega$ (where $\beta$ is an arbitrary scalar constant). Moreover by analy tic continuation, this implies

$$
\mathbf{E}^{T}\left(z^{-1}\right) \mathbf{E}(z)=\beta \mathbf{I}, \quad \text { for all } z
$$


Any transfer matrix satisfying (3) is called paraunitary or lossless $[6],[11],[13],[14]$. Under this condition, if we choose $\mathbf{R}(\mathbf{z})=z^{-n_{1}} \mathbf{E}^{T}\left(z^{-1}\right)$ then it is clear that perfect-reconstruction property (2) is satisfied. With this choice of $\mathbf{R}(z)$ it is easily verified that the synthesis filters are given by

$$
F_{k}(z)=d z^{-n_{0}} H_{k}\left(z^{-1}\right)
$$

where $d$ is a constant and $n_{0}$ is a positive integer. Clearly $F_{k}(z)$ are FIR filters of the same length as the FIR filters $H_{k}(z)$.

Paraunitariness of $\mathbf{E}(z)$ is not a necessary condition for obtaining perfect reconstruction structures. However, such matrices offer a simple means of achieving perfect reconstruction.

One way to constrain $\mathbf{E}(z)$ to be paraunitary is to obtain it as a cascade $K_{L-1} \Lambda_{L-2}(z) K_{L-2} \Lambda_{L-3}(z) \ldots$ $K_{2} \Lambda_{1}(z) K_{1}$ where $K_{m}$ are orthogonal matrices and $\Lambda_{m}(z)$ are diagonal matrices of delays (which are hence paraunitary). Each $M \times M$ orthogonal matrix $K_{m}$ is completely parameterized by a sequence of $M(M-1) / 2$ planar rotation angles [15]. If these angles are optimized such that the analysis filters $H_{k}(z)$ have good stopbands, then these filters can be used in QMF banks which will have perfect reconstruction property. Numerical design examples, and further theoretical results pertaining to necessary and sufficient conditions for PRF can be found in $[11]$.

THE TWO-BAND QMF LATTICE WITH PERFECT RECONSTRUCTION PROPERTY

The Smith-Barnwell result $[4]$ can be summarized as follows: let $G(z)$ be a linear-phase FIR filter of order $2(N-1)$ with frequency response $G\left(e^{j \omega}\right)=$ $e^{-j \omega(N-1)} G_{0}\left(e^{j \omega}\right)$ where $G_{0}\left(e^{j \omega}\right)$ is the amplitude response, which is a real and positive-valued quantity. Assume that $G(z)$ is a 'half-band' filter [11], so that the response exhibits a symmetry with respect to $\pi / 2$. Such symmetry implies, $G_{0}\left(e^{j \omega}\right)+G_{0}\left(e^{j(\omega+\pi)}\right)=$ constant for all $\omega$. In other words, $G(z)+(-1)^{N-1} G(-z)=$ $k z^{-(N-1)}$ where $k$ is a constant. (We can assume without loss of generality [11], that $N-1$ is odd). If we now define the analysis filter $H_{0}(z)$ to be any spectral factor of $G(z)$, and take the remaining filters in the 2 channel QMF bank to be

$$
\begin{gathered}
H_{1}(z)=z^{-(N-1)} H_{0}\left(-z^{-1}\right) \\
F_{0}(z)=z^{-(N-1)} H_{0}\left(z^{-1}\right), F_{1}(z)=z^{-(N-1)} H_{1}\left(z^{-1}\right)
\end{gathered}
$$

then the QMF bank exhibits perfect reconstruction property (PRF). In particular, we can verify that

$$
H_{0}\left(z^{-1}\right) H_{0}(z)+H_{1}\left(z^{-1}\right) H_{1}(z)=\text { constant }
$$

The pair $\left[H_{0}(z), H_{1}(z)\right\}$ will be called a perfect reconstruction pair (PRP for convenience).
Property (6a) implies that the FIR transfer functions $H_{0}(z)$ and $H_{1}(z)$ are power-complementary, i.e., the magnitude-squares add up to a constant:

$$
\left|H_{0}\left(e^{j \omega}\right)\right|^{2}+\left|H_{1}\left(e^{j \omega}\right)\right|^{2}=\text { constant }
$$

It has recently been established [6] that any pair of power complementary FIR transfer functions can be synthesized in the form of a cascaded lattice structure as shown in Fig. 3 (after normalizing the constant in (6b) to unity). If the further restriction in (5a) is imposed we can show that, the even numbered lattice coefficients satisfy $k_{2 m}=1$. This in turn means that, the lattice structure can be redrawn in the form of the analysis bank in Fig. 2(a). Since each criss-cross in Fig. 3 has the transfer matrix

$$
\left[\begin{array}{cc}
k_{m} & -\hat{k}_{m} \\
\hat{k}_{m} & k_{m}
\end{array}\right],
$$

it is orthogonal, hence the resulting $\mathbf{E}(z)$ is paraunitary. In other words, we can state the following result: if $H_{0}(z), H_{1}(z)$ are the analysis filters in the 2 channel Smith-Barnwell QMF bank, then the poly phase component matrix $\mathbf{E}(z)$ is necessarily paraunitary. Moreover, we can always implement the analysis bank in the form of a lattice structure. It can be verified that the synthesis filters giver in (5b) can be realized by transposing the analysis lattice. These lattice structures are shown in Fig. 4, which is clearly a special case of Fig. 2, for $M=2$. In Fig. 4, each lattice section has 2 multipliers unlike Fig. 3. This is because, we have divided through by $k_{m}$ in each section. Such denormalization does not affect any of the significant properites of our interest.

As a converse of the above result, we can show that, the transfer functions $H_{0}(z), H_{1}(z), F_{0}(z), F_{1}(z)$ generated by the lattice always satisfy (5) and (6), regardless of the values of $\alpha_{k}$. Accordingly, Fig. 4 represent a structurally perfect reconstruction system. In particular, the transfer function $H_{0}(z)$ generated by the lattice is necessarily a spectral factor of an appropriate linearphase FIR halfband filter with positive-valued amplitude response, and this statement holds for any values of $\alpha_{k}$.

If $H_{0}(z), H_{1}(z), F_{0}(z), F_{1}(z)$ are implemented in direct form, then due to coefficient quantization, the relation (6) does not hold exactly, and hence the reconstruction is not really perfect. On the other hand, if lattice structures are used for the actual implementaion of the QMF bank, (6) holds inspite of quantization of $\alpha_{k}$.

\section{OPTIMIZATION BASED ON THE LATTICE}

A nother advantage of the lattice-structre interpretation of the Smith-Barnwell result is in the design of the 
transfer function $H_{0}(z)$. One design procedure would be to compute a spectral factor of an appropriate FIR half band filter $G(z)$, which in turn can be designed using a standard method (such as the equiripple algorithm [4]). But the stopband attenuation of the halfband filter is about two times that of $H_{0}(z)$, and for large $N-1$ there are several zeros of $G(z)$ on the unit circle. Accordingly, spectral-factorization can be time consuming and/or ill-conditioned.

A second design procedure, based on the lattice structure of Fig. 4(a), relies on the fact that, the lattice transfer functions satisfy properties (5),(6) automatically. Accordingly, if we optimize the coefficients $\alpha_{k}$ so as to minimize the stopband energy of $H_{0}(z)$, the resulting transfer functions are ready for use in the $\mathrm{QMF}$ bank. A suitable objective function to minimize is

$$
\Phi=\int_{\omega_{S}}^{\pi}\left|H_{0}\left(e^{j \omega}\right)\right|^{2} d \omega
$$

Since (5a) holds, the stopband of $H_{1}(z)$ is an image of the stopband of $H_{0}(z)$. So minimizing (8) results in 'good' stopbands for both $H_{0}(z)$ and $H_{1}(z)$. Since (6b) also holds, the passband of $H_{0}(z)$ stays close to a fixed nonzero constant. In summary, minimizing (8) ensures good pass and stopbands for $H_{0}(z)$ and hence for $H_{1}(z)$.

Any pair of transfer functions $P_{m}(z), Q_{m}(z)$ in Fig. $4 \mathrm{a}$ is a PRP. When we optimize the coefficients $\alpha_{m}$ to minimize (8), the stopband attenuation of $P_{m}(z)$ increases as $m$ increases. In other words, in the optimized lattice, the purpose of each lattice section is to generate a better PRP $\left[P_{m+2}(z), Q_{m+2}(z)\right]$ from a given PRP $\left[P_{m}(z), Q_{m}(z)\right]$. As we progress towards the right in Fig. 4(a), we get better and better PRP's (and $\alpha_{m}$ gets smaller and smaller for large $m$ ). This is an important hierarchial or nesting property of the lattice: if we delete the rightmost lattice section, we do not destroy the perfect reconstruction property, but we decrease the available stop band attenuation of $H_{0}(z)$. Such a nesting property is of course not displayed by the direct form structure of a PRP.

As a design example, we designed a perfect reconstruction pair $\left[H_{0}(z), H_{1}(z)\right]$ of order $N-1=49$, with $\omega_{S}=0.6 \pi$. The resulting frequency response magnitudes are shown in Fig. $5(\mathrm{a})$. Note that $H_{0}(z)$ has 25 zeros on the unit circle, which is the maximum permissible for a spectral factor of any halfband filter of order $2(N-1)$. This feature was observed in all the examples we designed. Moreover, the minimum stopband attenuation of $H_{0}(z)$ was always very close to the equiripple designs obtainable by spectral factorization [4]. The peak attenuation is monotone decreasing rather than constant, and this is sometimes desirable [2]. Fig. 5(b) shows the magnitude response $\left|T\left(e^{j \omega}\right)\right|$ of the distortion function $T(z)=\hat{X}(z) / X(z)$. If the direct-form coeff- cients i.e., the impulse response of the resulting $H_{0}(z)$ is quantizied, $T(z)$ is not a delay any more; the resulting amplitude distortion is shown by the solid curve of the figure. For the lattice structure (broken lines), since $T(z)$ is a delay inspite of quantization, $\left|T\left(e^{j \omega}\right)\right|$ continues to be flat. This encourages us to think of implementing Fig. 4 with power of 2 coefficients, which can be optimized to minimize (8), while at the same time preserving PRF.

\section{References:}

[1] D. Esteban and C. Galand, "Application of quadrature mirror filters to split-band voice coding schemes," Proc. of the IEEE Int. Conf. on ASSP, Hartford, CN, pp. 191-195, May 1977.

[2] R. E. Crochiere and L. R. Rabiner, Multirate digital signal processing, Englewood Cliffs, NJ: Prentice Hall, 1983.

[3] C. R. Galand and H. J. Nussbaumer, "New quadrature mirror filter structures," IEEE Trans. on ASSP, pp. 522-531, June 1984.

[4] M. J. T. Smith and T. P. Barnwell III, "Exact reconstruction techniques for tree-structured subband coders," IEEE Trans. on ASSP., pp. 434-441, June 1986 (basic results presented at IEEE ICASSP 1984).

[5] K. Swaminathan and P. P. Vaidyanathan, "Theory and design of uniform DFT, parallel quadrature mirror filter banks," IEEE Trans. on Circuits and Systems, Dec. 1986.

[6] P. P. Vaidyanathan, "Passive cascaded lattice structures for low sensitivity FIR filter design, with applications to filter banks," IEEE Trans. on Circuits and Systems, pp. 1045-1064, Nov. 1986.

[7] J. H. Rothweiler, "Polyphase quadrature filters: a new subband coding technique," Proc. of the 1983 IEEE Int. Conf. on ASSP, pp. 1280-1283, Boston, March 1983.

[8] P. L. Chu, "Quadrature mirror filter design for an arbitrary number of equal bandwidth channels," IEEE Trans. on ASSP, pp. 203-218, Feb 1985.

[9] M. Vetterli, "Filter banks allowing for perfect reconstruction," in Signal Processing, Elsevier Science Publishers, North Holland, pp. 1-26, 1986.

[10] F. Mintzer, "Filters for distortion-free two-band multirate filter banks," IEEE Trans. on ASSP, pp. 626-630, June 1985

[11] P. P. Vaidyanathan, "Theory and design of Mchannel maximally-decimated quadrature mirror filters with arbitrary $M$, having perfect reconstruction property, Technical Report, California Institute of Technology, Pasadena, CA, April 1986 (also to appear in the IEEE Trans. ASSP, April 1987).

[12] M. J. T. Smith and T. P. Barnwell II, "A unifying 
framework for analysis/synthesis systems based on maximally decimated filter banks," Proc. IEEE Int. Conf. ASSP, Tampa, pp. 521-524, March 1985.

[13] P. P. Vaidyanathan and S. K. Mitra, "Low passband sensitivity digital filters: a generalized viewpoint and synthesis procedures," Proc. of the IEEE, pp. 404-423, April 1984.

[14] V. Belevitch, Classical Network Synthesis, HoldenDay, San Francisco, CA 1968.

[15] F. D. Murnaghan, The unitary and rotation groups, Spartan Books, 1962.

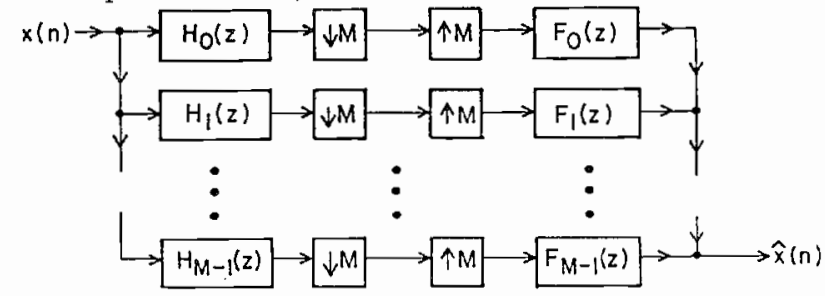

Fig. 1. The M-channel, maximally decimated Quadrature Mirror Filter bank.

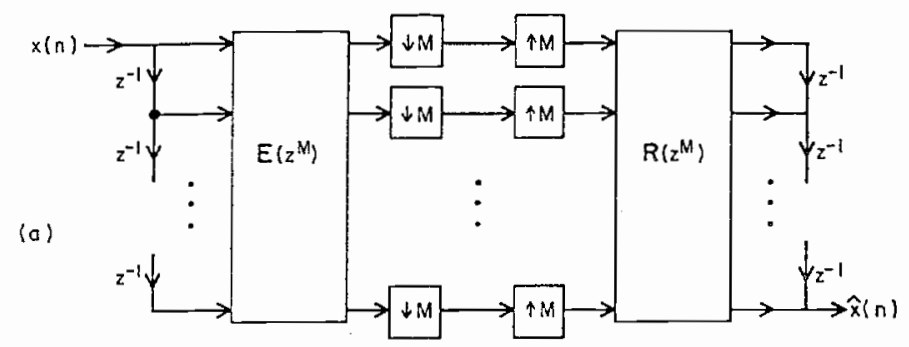

(b)

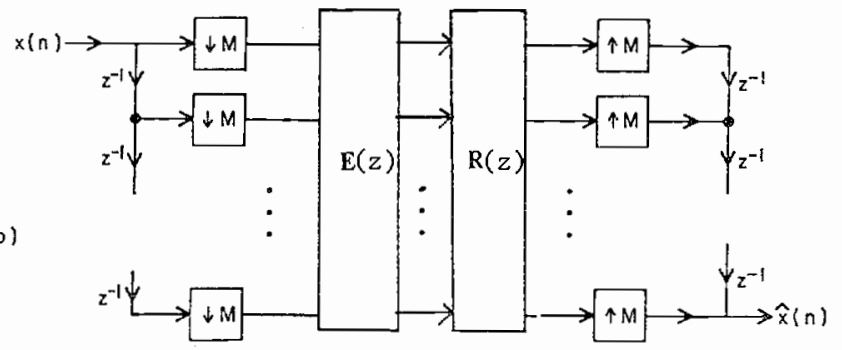

Fig. 2. Redrawing Fig. 1 in terms of the polyphase components.

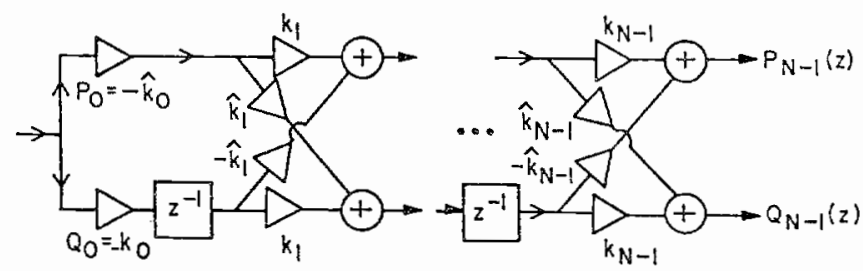

Fig. 3. Lattice structure for power-complementary FIR filters. Here $\mathrm{P}_{\mathrm{N}-1}(z)=\mathrm{H}_{0}(z), \mathrm{Q}_{\mathrm{N}-\mathrm{I}}(z)=\mathrm{H}_{1}(z)$.

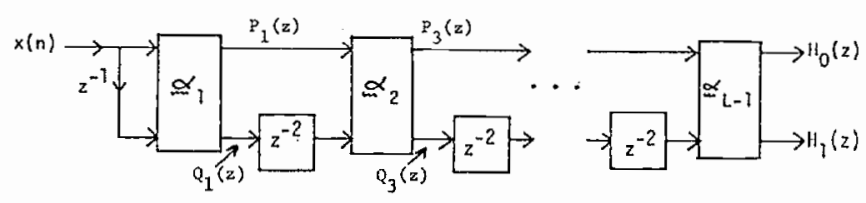

(a) Analysis bank implementation

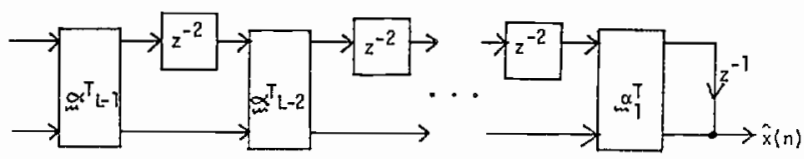

(b) Synthesis bank implementation

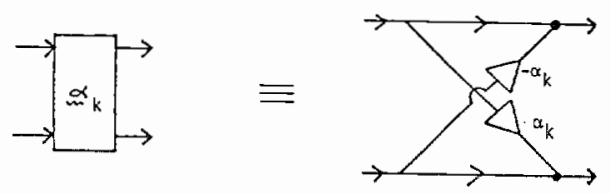

Fig. 4. Lattice structures for the QMF bank.
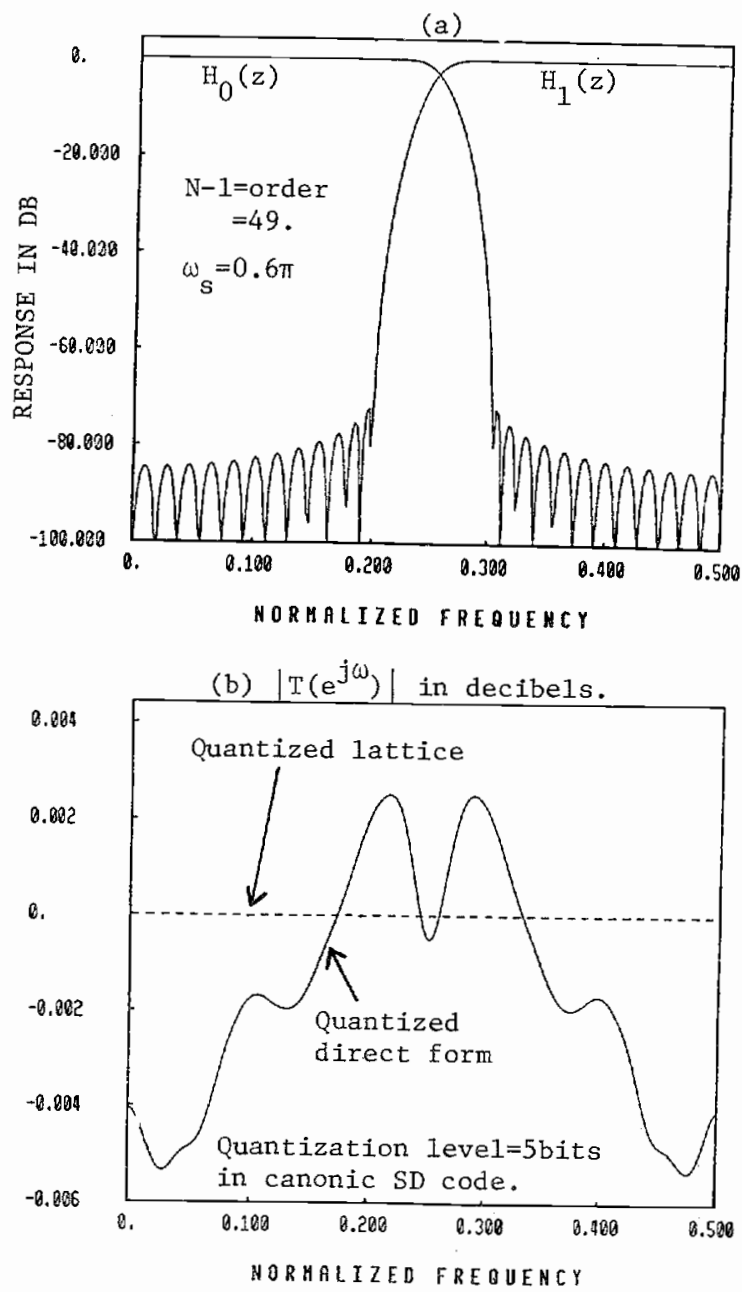

Fig. 5. Lattice based design example. 